\title{
Electronic engineering of a tetrathiafulvalene charge-transfer salt via reduced symmetry induced by combined substituents
}

Yasuhiro Kiyota, ${ }^{\mathrm{a}, \mathrm{b}}$ Ie-Rang Jeon, ${ }^{* \mathrm{~b}}$ Olivier Jeannin, ${ }^{\mathrm{b}}$ Maxime Beau, ${ }^{\mathrm{b}}$ Tadashi Kawamoto, ${ }^{\mathrm{a}}$ Pere Alemany, Enric Canadell, ${ }^{*}$ Takehiko Mori ${ }^{\mathrm{a}}$ and Marc Fourmigué*b

${ }^{a}$ Department of Materials Science and Engineering, Tokyo Institute of Technology, O-okayama 2-12-1, Meguro-ku, Tokyo 1528552, Japan.

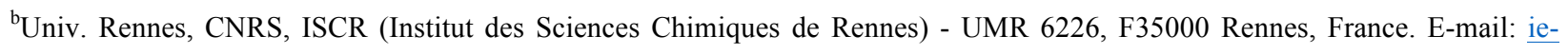
rang.jeon@univ-rennes1.fr, marc.fourmigue@univ-rennes1.fr

${ }^{\circ}$ Departament de Ciència de Materials i Química Física and Institut de Química Teòrica i Computacional (IQTCUB), Universitat de Barcelona, Martí i Franquès 1, Barcelona 08028, Spain.

${ }^{\mathrm{d} I n s t i t u t ~ d e ~ C i e ̀ n c i a ~ d e ~ M a t e r i a l s ~ d e ~ B a r c e l o n a ~(C S I C), ~ C a m p u s ~ d e ~ l a ~ U A B, ~ E-08193 ~ B e l l a t e r r a, ~ S p a i n . ~ E-m a i l: ~ c a n a d e l l @ i c m a b . e s ~}$

A 1:1 metallic charge-transfer salt is obtained by cosublimation of $(Z, E)-(\mathrm{SMe})_{2} \mathrm{Me}_{2} \mathrm{TTF}$ and TCNQ. Xray diffraction studies confirm that the formation of segregated stacks comprising donor and acceptor molecules in $\left[(E)-(\mathrm{SMe})_{2} \mathrm{Me}_{2} \mathrm{TTF}\right](\mathrm{TCNQ})$. The crystal packing features lateral $\mathrm{S} \cdots \mathrm{S}$ interactions between TTF stacks, which is in sharp contrast to that in (TTF)(TCNQ). Structural analysis and theoretical studies afford a partial charge-transfer $(\boldsymbol{X} \approx 0.52$ ), leading to a system with the electronic structure close to quarter-filled. Resistivity measurements reveal that this material behaves as a metal down to $56 \mathrm{~K}$ and 22 $\mathrm{K}$ at $1 \mathrm{bar}$ and $14.9 \mathrm{kbar}$, respectively. The thermopower is negative in the metallic regime, indicating the dominant role of the acceptor stacks for the observed conducting behavior. Analysis of a single-crystal EPR spectra shows the remaining spin susceptibility at $4.3 \mathrm{~K}$, suggesting the importance of the Hubbard $\mathrm{U}$ correction. These results highlight the judicious engineering of electronic and geometrical effects on the TTF core; the combined use of methyl and thiomethy groups has decreased the TCNQ bandwidth while keeping the segregated stacks, converting the metal to insulator (M-I) transition more $4 k_{\mathrm{F}}$ like. In addition, the enhanced $\mathrm{S} \cdots \mathrm{S}$ contacts between the TTF stacks lead to more rapidly decreasing M-I transition temperature under pressures.

\section{Introduction}

The discovery of metallic behaviour in crystalline organic molecular materials has stimulated a plethora of different research areas covering chemistry and physics. ${ }^{1}$ The most notable example is the charge transfer donor-acceptor compound, $(\mathrm{TTF})(\mathrm{TCNQ}) \quad(\mathrm{TTF}=$ tetrathiafulvalene; $\mathrm{TCNQ}=7,7,8,8$ tetracyanoquinodimethane), which was the first organic conductor to present a metallic conductivity in a 
wide temperature range down to $59 \mathrm{~K} .{ }^{2}$ This compound features an anisotropic crystal structure where donor and acceptor molecules form segregated stacks which facilitate a good overlap of respectively HOMOs and LUMOs $\pi$-type frontier orbitals along the stack direction to render a $1 \mathrm{D}$ electronic structure. ${ }^{3}$ The accumulated knowledge on such a system emphasizes the critical role of structural chemistry and crystal engineering. ${ }^{4}$ In these molecular systems, a subtle change in chemical structure is known to drastically modify geometrical and electronic structures, which in turn enable to discover new exotic physical phenomena and to underpin physical understanding. ${ }^{5}$ It is particularly interesting that the intriguing interplay of $2 k_{\mathrm{F}}$ and $4 k_{\mathrm{F}}$ instabilities have been extensively investigated in (TTF)(TCNQ). ${ }^{4}$

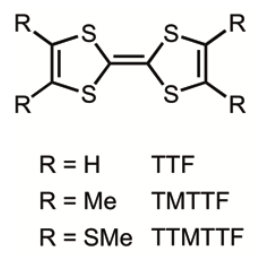

$(E)-1$

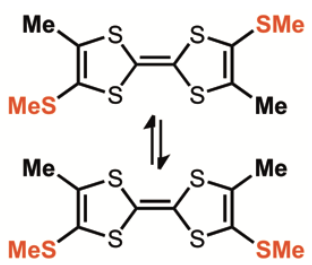

Scheme 1. Tetrathiafulvalene derivatives with different substituents.

It has been known that tetramethyl-TTF (TMTTF) as well as hexamethylene-TTF (HMTTF) stabilize the metallic conduction, ${ }^{12}$ whereas tetrathiomethyl-TTF (TTMTTF) makes insulating TCNQ complexes due to the mixed-stack structures in DDA and DDAA sequences. ${ }^{13 a}$ This comes from the strong dimerization of TTMTTF associated with the steric hindrance of the out-of-plane thiomethyl groups. Methyl substitution increases the donor ability, but thiomethyl substitution reduces the donor ability (Figure S5). Therefore, in order to finely control the electronic structure without destroying the segregated stacks, we have brought our attention to a TTF donor molecule with two thiomethyl- and two methyl-substituents (Z,E)$(\mathrm{SMe})_{2} \mathrm{Me}_{2} \mathrm{TTF},(Z, E)-\mathbf{1}$ (Scheme 1).

At variance with classical TTF, TMTTF, TTMTTF and BEDT-TTF (bis(ethylenedithio)-tetrathiafulvalene) molecules with $D_{2 \mathrm{~h}}$ symmetry, such systems can exist into $Z\left(C_{2 \mathrm{v}}\right)$ and $E\left(C_{2 \mathrm{~h}}\right)$ forms, which easily interconvert in solution upon oxidation, making often difficult their (electro)crystallization ${ }^{6}$ despite their readily accessible synthesis. Such a difficulty is well demonstrated by the scarcity of the structurally characterized donor-acceptor two-chain charge transfer salts comprising such $Z$ - or $E$-TTF isomers. ${ }^{7}$ Our specific interest for 1 also comes from its first oxidation potential $\left(E_{1 / 2}=+0.41 \mathrm{~V} v s\right.$. SCE, Figure S1) close to that of TTF itself $(+0.33 \mathrm{~V})$, a consequence of the electron-rich methyl groups which compensate in part the electron withdrawing effect of the thiomethyl groups. We postulate that such a close proximity of electrochemical potentials likely triggers a non-zero charge transfer if this donor is associated with TCNQ, ${ }^{8}$ as demonstrated below. 


\section{Results and discussion}

\section{Co-crystallization and structural analyses}

The neutral $(Z, E)$-1 was prepared according to the modified literature procedure, by successive deprotection of $(Z, E)-\left(\mathrm{SCH}_{2} \mathrm{CH}_{2} \mathrm{CN}\right)_{2}(\mathrm{Me})_{2}$ TTF with $\mathrm{CsOH}$ and further alkylation with MeI. ${ }^{9}$ Cosublimation of $(Z, E)-1$ and TCNQ in a two-zones furnace ${ }^{10}$ under vacuum afforded black, flake-shaped crystals of $[(E)-1](T C N Q)$ in 3 weeks. In addition to $[(E)-1](T C N Q)$, we have also observed the deposition of orange needle-shaped crystals of the donor molecules and yellow flake-shaped crystals of the acceptor molecules within the cosublimation tube (see Experimental section). NMR analysis on the orange crystals that remained after the sublimation indicated that it only contains $E$-isomer (Figure S2). This observation strongly suggests that the $Z$-isomer converts to $E$-isomer during the vaporization in the presence of the TCNQ vapor, and therefore, exclusively affords $[(E)-\mathbf{1}](\mathrm{TCNQ})$ as a co-crystallized product. Please note that the sublimation of $(Z, E)$ 1 without TCNQ afforded the mixture of $Z$ - and $E$-isomers (Figure S2). To the best of our knowledge, such a discrimination of isomers using co-sublimation has never been reported. It is worh to mention that the solution diffusion method for co-crystallization was not successful to obtain single crystals for further analysis, although it provided microcrystalline powders consisting of $[(E)-\mathbf{1}](\mathrm{TCNQ})$, as indicated by the powder X-ray diffraction (figure S3).

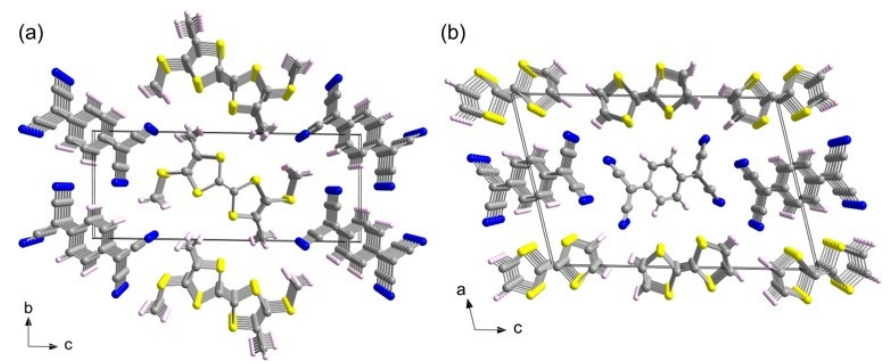

Figure 1. Projection views of the crystal packing in $[(E)-1](T C N Q)$ (a) and (TTF)(TCNQ) (b). Yellow, blue, gray, and pink spheres represent $\mathrm{S}, \mathrm{N}, \mathrm{C}$, and $\mathrm{H}$ atoms, receptively.

The compound crystallizes in the triclinic system, $P-1$ space group, with both $(E)-\mathbf{1}$ and TCNQ molecules on an inversion centre. The structure consists of segregated stacks of donor and acceptor molecules running both along the crystallographic $a$ axis, and alternating along the $c$ axis, as shown in Figure 1a.

The intermolecular overlaps along the stacks adopt the bond-over-ring motif commonly observed in highly conducting compounds, with plane-to-plane distances of 3.474 and $3.227 \AA$ in the stacks of $(E)-1$ and TCNQ respectively. Note that these values are larger than those reported in the RT structure of (TTF)(TCNQ) (3.451 and $3.188 \AA$ respectively) (Table S1), ${ }^{11}$ an indication of possibly weaker intermolecular interactions along the stacks in $[(E)-1](T C N Q)$. In the crystallographic ac plane (Figure S4), 
all molecules are tilted in the same direction with respect to the (100) plane, with an angle of 22.23 and $31.65^{\circ}$ for $(E)-1$ and TCNQ respectively, leading to an interplanar dihedral angle of $9.42^{\circ}$. A remarkable feature is the relative arrangement of the stacks which allows direct $\mathrm{S} \cdots \mathrm{S}$ inter-stack contacts along the $b$ axis (Figures 1a and S4) while such lateral interactions are absent in (TTF)(TCNQ) (Figure 1b). Taken together, the arrangement and the packing of donor and acceptor molecules in [(E)-1](TCNQ) strongly differs from that of the unsubstituted (TTF)(TCNQ). ${ }^{11}$

Analogous 1:1 salts were also reported with the $D_{2 \mathrm{~h}}$-symmetry TTFs such as TMTTF and TTMTTF. The (TMTTF)(TCNQ) metallic salt crystallizes in the $P 2 / c$ space group with stacks of TMTTF molecules interacting also sideways, but with large and opposite tilt angles $v s$. the stacking axis. ${ }^{12}$ The (TTMTTF)(TCNQ) charge transfer complex is an insulating compound with mixed stacks, as explained in Introduction. ${ }^{13}$ Although tetraethyl-TTF (TETTF) has a similar chemical potential located near TCNQ (Figure S5), it also makes mixed stacks with TCNQ, (TETTF)(TCNQ) 2 , owing to the out-of-plane ethyl groups. ${ }^{13 \mathrm{~b}}$ However, DEDMTTF forms segregated stacks in the TCNQ complex, ${ }^{13 \mathrm{c}}$ where the terminal methyl moieties are within the same plane as the TTF part but extend to side of the TTF. The resulting molecular packing of (DEDMTTF)(TCNQ) is very similar to the present complex (Figure 1a), and has direct contacts between the TTF stacks. These comparisons indicate that the balanced substitution between $-\mathrm{SMe}$ and $-\mathrm{Me}$ groups in the present donor $\mathbf{1}$ indeed allows for an original structure which enhances the structural dimensionality of the conducting sheets while avoiding DADA mixed stacks.

To estimate the degree of charge transfer, the bond distances within TCNQ were carefully examined using Kistenmacher's relationship (Table S2 and Figure S6). ${ }^{14}$ The calculated charge of TCNQ is 0.52 , implying a nearly quarter-filled band system. Note that this value is also coherent with $\rho=0.59$ estimated from the $\mathrm{C} \equiv \mathrm{N}$ stretch of the FT-IR spectrum ${ }^{15}$ and the theoretical calculations (vide-infra).

\section{Transport properties}
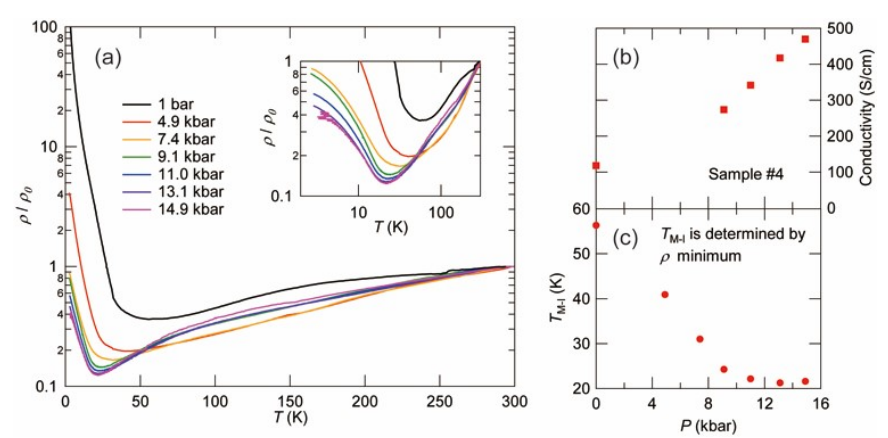
Figure 2. (a) Temperature dependence of the resistivity under pressure. The inset is a logarithmic plot for clearly at low temperatures. Pressure dependence of (b) the room-temperature conductivity and (c) $T_{\mathrm{M}-\mathrm{I}}$ determined by the resistivity minimum.

The variable-temperature electrical resistivity was measured under different pressures on single crystals along the $a$ stacking axis (Figure 2). At $300 \mathrm{~K}$, the conductivity is $118 \mathrm{~S} / \mathrm{cm}$ under ambient pressure, which is comparable to the known organic charge transfer conducting compounds. ${ }^{3}$ As temperature decreases, the resistivity decreases smoothly to reach a minimum value at $56 \mathrm{~K}$ before an abrupt increase, indicating the occurrence of a metal to insulator (M-I) transition. The activation energy in the insulating region is estimated at $E_{\mathrm{a}}=31 \mathrm{meV}$. Before the M-I transition, the resistivity drop shows a deviation around $130 \mathrm{~K}$, which is confirmed by the maximum of the derivative (Figure S7), likely related to the electronic instability in either the donor or the acceptor stacks (vide-infra).

Upon increasing pressure, the room-temperature conductivity increases and the M-I transition temperature significantly shifts to lower temperatures, to reach $470 \mathrm{~S} / \mathrm{cm}$ and $22 \mathrm{~K}$, respectively, at $14.9 \mathrm{kbar}$ (Figures $2 \mathrm{~b}$ and $2 \mathrm{c}$ ). At this highest available pressure, the resistivity rise shows no saturation and $E_{\mathrm{a}}$ decreases by half $\left(E_{\mathrm{a}}=16 \mathrm{meV}\right)$. This is in stark contrast with (TTF)(TCNQ), where the conductivity increases less than twice and the M-I transition temperature hardly varies under similar pressures. ${ }^{3 a, 16}$ The comparatively large pressure-sensitivity of the present compound is likely attributable to the enhanced structural dimensionality coming from the side-by-side $\mathrm{S} \cdots \mathrm{S}$ contacts. The applied pressure may indeed reinforce the inter-stack contacts which might ultimately lead to the suppression of the electronic Peierls instability expected for a purely $1 \mathrm{D}$ electronic system.

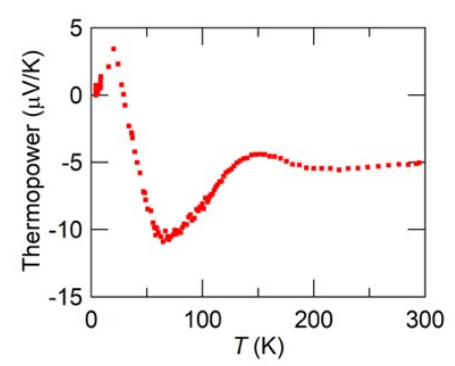

Figure 3. Variable-temperature thermopower of $[(E)-\mathbf{1}](\mathrm{TCNQ})$.

As shown in Figure 3, the thermopower exhibits small negative values over the metallic region, indicating that the transport is dominated by the acceptor stacks. ${ }^{17}$ However, in contrast to (TTF)(TCNQ) where it amounts to $-40 \mu \mathrm{V} / \mathrm{K}$ at $300 \mathrm{~K}$, the small absolute value for $[(E)-1](\mathrm{TCNQ}),-5 \mu \mathrm{V} / \mathrm{K}$, indicates an increased contribution of the donor stacks to the conductivity. Upon lowering temperature, the thermopower 
shows a small peak around $150 \mathrm{~K}$ then a gradual decrease. Given the negative carriers become more dominant below $150 \mathrm{~K}$, the anomaly at $150 \mathrm{~K}$ is probably to be associated with the setting of $4 \mathrm{kF}$ instability in the donor stack. Note that such a peak was observed also for (TMTTF)(TCNQ) ${ }^{17}$ Below $70 \mathrm{~K}$, the strong upturn of the thermopower is observed, which may be due to the setting of modulation in the TCNQ stacks that finally condense and open a gap around $50 \mathrm{~K}$.

\section{ESR analyses}

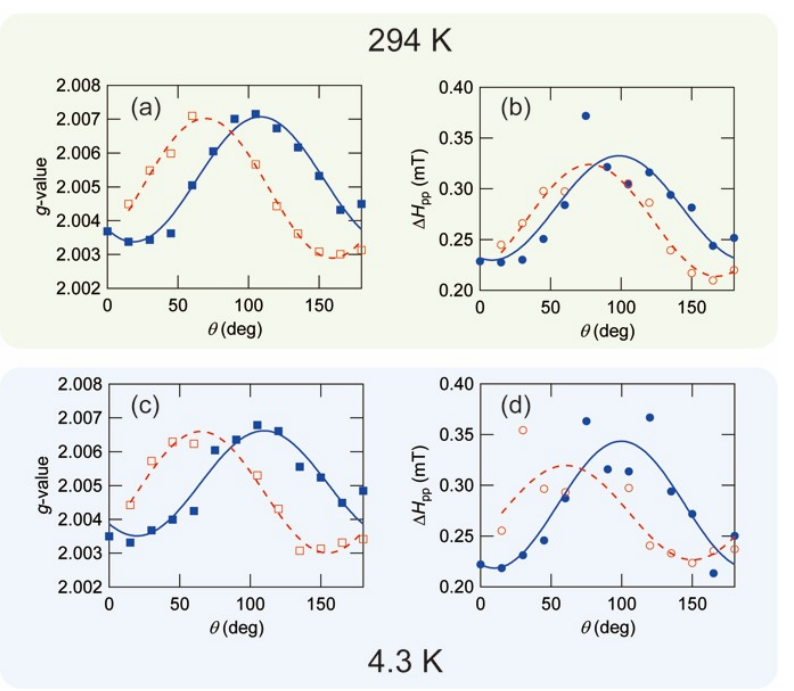

Figure 4. Angle dependence of the $g$-value and the linewidth at (a)(b) $294 \mathrm{~K}$ and (c)(d) $4.3 \mathrm{~K}$, highlighting two independent Lorentzians indicated with red (open) and blue (filled) symbols.

Figures 4 shows the angle dependence of the extracted g-values and linewidths at room temperature and at 4.3 K. Although single crystals were chosen carefully, the observed ESR signal was systematically composed of two Lorentzians (Figure S8). ${ }^{18}$ Given that the two Lorentzians manifest at the same angles in all three samples investigated, this is likely an inherent property of the compound and the splitting stems from the existence of inversely tilted domains, which have borders in the $a b$ plane (Figure S9). In view of the extremely small deviation from the monoclinic cell $\left(\beta=90.292^{\circ}\right)$, this seems to be inherently associated with the present crystal.

Figure 5 shows temperature dependence of the spin susceptibility and the linewidth estimated from a single Lorentzian at $0^{\circ}$ and double Lorenzian at $30^{\circ}$. The spin susceptibility is almost constant with a slight decrease down to $65 \mathrm{~K}$, indicating the Pauli-paramagnetic like behavior. Below $65 \mathrm{~K}$, the spin susceptibility gradually increases. The linewidth also takes a minimum at $65 \mathrm{~K}$ and then increases. The observed similar tendency for both Lorentzians supports the same origin of the two signals at $30^{\circ}$. Broadening of the ESR signal is likely related to the nesting of the TCNQ Fermi surface as observed in related compounds, (TTF)(TCNQ) and (TMTTF)(TCNQ). ${ }^{18,19}$ At low temperature, the spin susceptibility remains non-zero 
even at $4.3 \mathrm{~K}$, indicating charge-spin decoupling in the compound. Note that (DEDMTTF)(TCNQ) seems to remain paramagnetic down to low temperatures as well. ${ }^{13 c}$

\section{Electronic structure calculation}
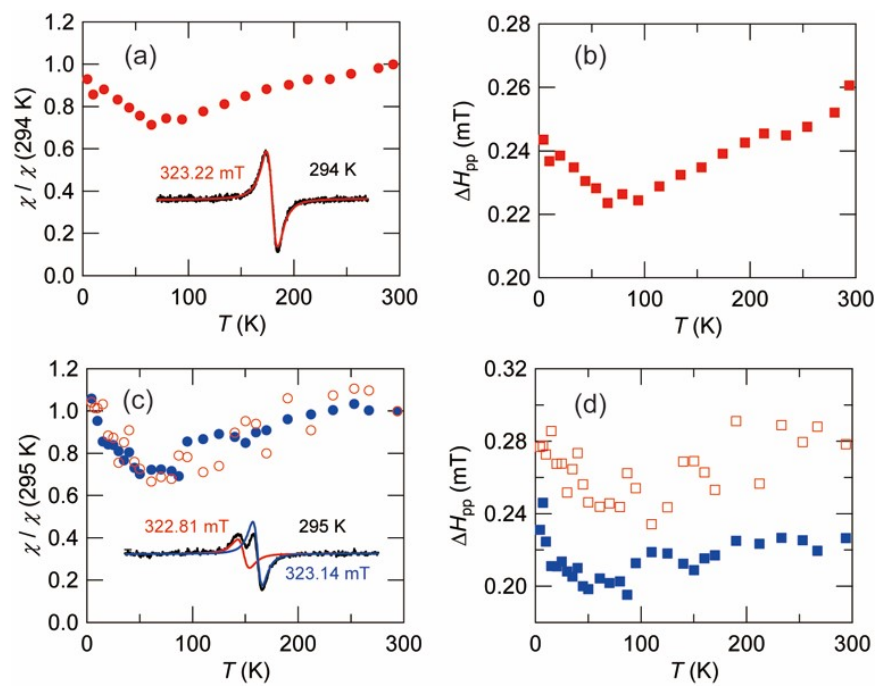

Figure 5. Temperature dependence of the spin susceptibility and the linewidth for (a)(b) the single Lorentzian at $0^{\circ}$ and for (c)(d) the double Lorentzians at $30^{\circ}$. The two independent Lorentzians designated with a red (open) symbol and a blue (filled) symbol.

The electronic structure of $[(E)-1](T C N Q)$ was determined from first-principles $\mathrm{DFT}+\mathrm{U}$ (U being an effective Hubbard correction term ${ }^{20}$ ) calculations based on the $150 \mathrm{~K}$ crystal structure. Although DFT calculations usually provide a good description of the electronic structure of molecular conductors, twochain molecular conductors like the present system still face us with some noticeable problems. For instance, the calculated charge transfer in TTF-TCNQ is $\sim 0.72-0.74$ irrespective of the particular functional and basis set used ${ }^{21,22}$ whereas the experimental estimations are $0.55-0.59 .{ }^{4}$ As we have recently discussed, ${ }^{22}$ this problem mostly concerns two-chain conductors containing TTF or TTF-based donors because of a deficient description of their HOMO bands.

These bands are usually found to be wider than the experimental estimations in two-chain systems for which $4 \mathrm{k}_{\mathrm{F}}$ fluctuations associated with the TTF-based component have been observed. These observations mean that electronic repulsions in the TTF-based chains are important and that they are underestimated by the DFT approach. The simplest way to remedy for this drawback is by using an effective Hubbard correction term for S 3p orbitals which contracts them and leads to narrower HOMO bands and smaller charge transfers. ${ }^{22}$ Since the present system contains TTF-based chains we have considered the U dependence of the electronic parameters of TTF-TCNQ, HMTTF-TCNQ and TMTTF-TCNQ (see for instance Figures $\mathrm{S} 10$ to $\mathrm{S} 11$ ) and found that a value $\mathrm{U}=6 \mathrm{eV}$ leads to reasonable results for band widths and charge transfers. 
For instance, using their room temperature crystal structure the calculated vs. experimental charge transfers (determined at $25 \mathrm{~K}$ ) ${ }^{4}$ for TTF-TCNQ, TMTTF-TCNQ and HMTTF-TCNQ are 0.57/0.59, 0.56/0.57 and $0.68 / 0.72$. Thus, we have adopted this value in our study.

The calculated band structure and density of states are reported in Figure 6 . The upper band at $\Gamma$ is based on the (E)-1 HOMO and the lower band is based on the TCNQ LUMO. The two bands cross along the intrachain $\Gamma-X$ line, at the Fermi energy. However, in these two-chain conductors the avoided crossing between the HOMO and LUMO bands is usually slightly overestimated by DFT so that we believe that the gap along $\Gamma-\mathrm{X}$ is smaller and probably irrelevant for most temperatures. The small but non nil dispersion along the interchain $b^{*}$-direction $(\Gamma-\mathrm{Y})$ of the donor band, which is associated with an $\mathrm{S} \cdots \mathrm{S}$ contact of $3.767 \AA$, slightly lowers the energy of the upper band and consequently overlaps with the top of the lower band, definitely leading to a band overlap inside the Brillouin zone and thus, a metallic-type behavior.
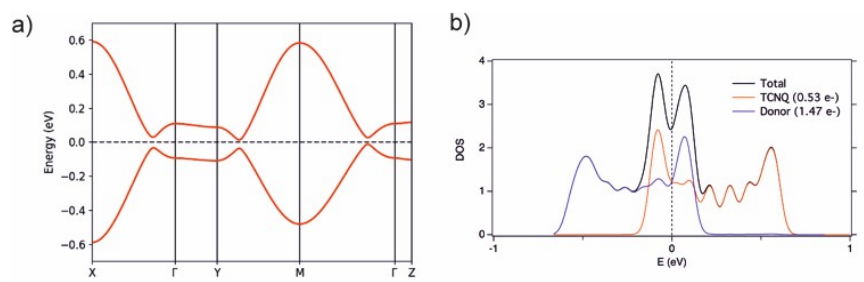

Figure 6. Calculated band structure (a) and Density of states (b) for $\mathrm{Me}_{2}(\mathrm{SMe})_{2} \mathrm{TTF}-\mathrm{TCNQ}$ at $150 \mathrm{~K}$. The Fermi level is the energy zero. $\Gamma=(0,0,0), \mathrm{X}=(1 / 2,0,0), \mathrm{Y}=(0,1 / 2,0), \mathrm{M}=(1 / 2,1 / 2,0)$ and $\mathrm{Z}=(0,0,1 / 2)$ in units of the triclinic reciprocal lattice vectors. The DOS is given in units of electrons per $\mathrm{eV}$ and per formula unit.

The avoided band crossing occurs for a $k_{\mathrm{F}}$ value of $0.13 a^{*}$ and consequently, the charge transfer $\left(\rho=4 \times k_{\mathrm{F}} / \mathrm{a}^{*}\right)$ is 0.52 . Integration of the DOS curves (see Figure $6 \mathrm{~b}$ where the total DOS as well as the separate contributions of the donor and acceptor bands are also shown) leads to $\rho=0.53$. These values are very similar to the $\rho=0.52$ one estimated above from the bond lengths of the acceptor thus confirming that the charge transfer must be very close to 0.5 . This situation is clearly different from that of the archetypal (TTF)(TCNQ) for which the charge transfer $(\rho=0.59)$ is definitely far from the quarterfilled/empty case and thus a different physical behavior may be expected. Note that whatever the value of $\mathrm{U}$ used in the calculations, the difference with (TTF)(TCNQ) does not practically change (Figure S10) so that the result is a robust one.

\section{Comparison with (TTF)(TCNQ)}

What is the origin of the charge transfer difference with (TTF)(TCNQ)? It may be argued that it is due to the decrease in the HOMO energy induced by the -SMe substituents, illustrated by the increase of first 
oxidation potential when compared with TTF. However, the charge transfer in these two-chain molecular conductors subtly depends on both electronic and structural factors. A decrease of the charge transfer can also occur if one or both of the HOMO and LUMO bands become narrower. The dispersion of the TCNQ band depends essentially of the $\mathrm{C} \cdots \mathrm{C}$ contacts along the intrachain $a$ direction. As mentioned above, these contacts in $[(E)-\mathbf{1}](\mathrm{TCNQ})$ (Table S1) are clearly longer than even those for the RT structure of (TTF)(TCNQ). Hence we can conclude that the TCNQ band in the present salt must be narrower than in (TTF)(TCNQ).

The dispersion of the donor band depends essentially from the six intra-stack $\mathrm{S} \cdots \mathrm{S}$ contacts. In the RT structure of TTF-TCNQ, the same contacts are again found to be shorter (Table S1), as evidenced also by the cell parameter along the chains direction $(3.842 \AA$ for $[(E)-1](T C N Q)$ at $150 \mathrm{~K}$ vs $3.819 \AA$ for (TTF)(TCNQ) at RT). However, the (E)-1 donor has two additional sulfur atoms involved in the HOMO. The extra $\mathrm{S}_{\mathrm{SMe}}{ }^{\cdots} \mathrm{S}_{\mathrm{TTF}}$ intra-stack contacts increase the HOMO $\cdots$ HOMO interaction with respect to (TTF)(TCNQ) and it is not clear if, in the present compound, the dispersion of the HOMO band should slightly decrease or even increase.

Assuming a linear relationship for the thermal evolution of the DFT band widths, we estimate that the widths of the HOMO and LUMO bands for (TTF)(TCNQ) at $150 \mathrm{~K}$ should be 0.59 and $0.83 \mathrm{eV}$, respectively. The calculated band widths for the present compound are 0.70 and $0.68 \mathrm{eV}$, respectively. As expected, the band width for the TCNQ band decreases in $[(E)-1](T C N Q)$. In contrast, the decrease in the strength of the intrachain $\mathrm{S} \cdots \mathrm{S}$ interactions of the TTF core is more than compensated by the extra $\mathrm{S}_{\mathrm{SMe}} \cdots \mathrm{S}_{\mathrm{TTF}}$ contacts, leading ultimately to an increase of the HOMO band width. However, we note that the variation for the TCNQ band is somewhat larger. In addition, the increase in the donor band width (and thus, of charge transfer) will mostly compensate the intrinsic lowering in the HOMO energy (and thus, the decrease of the charge transfer) due to the-SMe substituents. We thus suggest that the substantial decrease of the $\mathrm{C} \cdots \mathrm{C}$ contacts in the TCNQ stacks is the main reason behind the small but significant charge transfer decrease, now very close to the quarter-filled/empty value.

\section{Low temperature behaviour scenario}

What are the possible scenario for the low temperature behavior of this compound? An important factor to consider is the filling of the LUMO/HOMO bands which at low temperatures may become almost exactly quarter-filled/empty $(\rho=0.5)$ or can keep a slightly different value. In view of the transport measurements reported above and relying on analogies with other TTF-based two-chain salts ${ }^{4,17}$ we suggest the following scenario. The small anomaly occurring around $150 \mathrm{~K}$ clearly seen in the thermopower measurements is probably to be associated with the setting of $4 k_{\mathrm{F}}$ fluctuations in the donor stacks since the negative carriers become more dominant below $150 \mathrm{~K}$. The strong upturn around $70 \mathrm{~K}$ may be due to the setting of a 
fluctuations regime in the TCNQ stacks which finally condense and open a gap at around $50 \mathrm{~K}$. However, if a charge transfer very near to the quarter-filled/empty case remains at low temperatures the modulation on the TCNQ stacks could be a $2 k_{\mathrm{F}}$ tetramerization and the regime change in the $(E)-\mathbf{1}$ stacks could then be of the $4 k_{\mathrm{F}}$ charge-ordering or Mott-dimer types, explaining that the magnetic susceptibility remains nonzero until very low temperatures. ${ }^{23} \mathrm{X}$-ray diffuse scattering studies are needed in order to shed light on these issues.

\section{Conclusions}

In this study, we have successfully grown a 1:1 co-crystal of $(E)-\mathbf{1}$ and TCNQ and have investigated its physical properties in detail. The obtained crystal of $[(E)-1](T C N Q)$ features the segregated $\mathrm{D}$ and A stacks with the DA packing that is notably different when compared with the analogous charge transfer TCNQ salts comprising the classical TTF donors. In particular, the lateral $\mathrm{S} \cdots \mathrm{S}$ contacts between donor stacks give rise to the structural two-dimensionality in $[(E)-1](T C N Q)$.

The band structure of the compound features close to a quarter-filled system with $\rho \approx 0.52$, leading to a metallic behaviour down to $56 \mathrm{~K}$. Moreover, both the conductivity and the metal-to-insulator transition temperature are significantly influenced by the applied pressure, likely due to the enhanced structural dimensionality.

In particular, slight delocalization in the HOMO of the donor by means of the two thiomethyl- and two methyl-substituents leads to a situation where the donor and acceptor band widths are very similar and the charge transfer is very near the commensurate value 0.5 . To the best of our knowledge the only other twochain salts of this family exhibiting both features are (DEDMTTF)(TCNQ) and (TMTTF)(DMTCNQ) (DMTCNQ $=$ 2,5-dimethyl-7,7,8,8-tetracyanoquinodimethane). ${ }^{4,13 \mathrm{c}}$ These salts lie just at the borderline between those where the dominant structural instability changes from $2 k_{\mathrm{F}}$ to $4 k_{\mathrm{F}}{ }^{4}$ Thus, it would be extremely interesting to study if this is also the case for the present salt. If it is indeed the case, small structural or electronic modifications could bring substantially different physical behaviors and provide invaluable information to settle the difficult issue of the competition between structural instabilities in twochain conductors.

Work is underway to prove the ground state of $[(E)-\mathbf{1}](\mathrm{TCNQ})$ and to explore the promising role of $(Z, E)$ $\mathbf{1}$ as electron donor to grow co-crystals with intriguing electronic structures.

\section{Experimental}

\section{General methods}

Chemicals and materials from commercial sources were used without further purification. Solvents were dried using a commercial solvent purification system from Inert. The $(Z, E)-\left(\mathrm{SCH}_{2} \mathrm{CH}_{2} \mathrm{CN}\right)_{2}(\mathrm{Me})_{2} \mathrm{TTF}$ was 
prepared according to the literature procedure. ${ }^{9}$ Melting points were measured on a Kofler hot-stage apparatus. NMR spectra were recorded on a Bruker AV III $300 \mathrm{MHz}$ NMR spectrometer at room temperature using $\mathrm{CDCl}_{3}$. Column chromatography was performed using Teledyne Isco CombiFlash ${ }^{\circledR}$ Rf400. Cyclic voltammetry measurements were carried out in a one-compartment cell under argon, equipped with a platinum wire, a glassy carbon, and a Saturated Calomel Electrode (SCE) as working, counter, and reference electrodes, respectively, using a Bio-Logic SP-50 potentionstat. Analyte solutions $\left(10^{-3} \mathrm{M}\right.$ solution of complex) were prepared in $0.1 \mathrm{M}$ solutions of $\left(\mathrm{NBu}_{4}\right)\left(\mathrm{PF}_{6}\right)$ in $\mathrm{CH}_{2} \mathrm{Cl}_{2}$.

\section{Synthesis of $(Z, E)-1$}

To a solution of the $(Z, E)-\left(\mathrm{SCH}_{2} \mathrm{CH}_{2} \mathrm{CN}\right)_{2}(\mathrm{Me})_{2} \mathrm{TTF}(300 \mathrm{mg}, 0.75 \mathrm{mmol})$ in $75 \mathrm{~mL}$ of THF was added under argon a solution of $\mathrm{CsOH} \cdot \mathrm{H}_{2} \mathrm{O}(372 \mathrm{mg}, 2.22 \mathrm{mmol})$ in $10 \mathrm{~mL}$ of $\mathrm{MeOH}$ dropwise at $0{ }^{\circ} \mathrm{C}$. The resulting solution was stirred at $25^{\circ} \mathrm{C}$ for 90 minutes to afford a dark mixture. MeI $(0.3 \mathrm{~mL}, 4.82 \mathrm{mmol})$ was then added to the flask, and the resulting mixture was stirred for $12 \mathrm{~h}$ to give a yellow solution. After quenching the reaction with $60 \mathrm{~mL}$ of distilled $\mathrm{H}_{2} \mathrm{O}$, the organic solvent mixture was removed from the reaction under vacuum, and the resulting residue was extracted into $\mathrm{CH}_{2} \mathrm{Cl}_{2}(4 \times 60 \mathrm{~mL})$. The organic extracts were dried over magnesium sulfate, filtered, and then concentrated under reduced pressure to give a red oil. The crude oil was purified by flash column chromatography on a silica gel to afford $(Z, E)-1$ as an orange solid (198 mg, $82 \%$ ). The product was recrystallized by slowly evaporating the saturated EtOAc solution. Column conditions: silica cartridge $12 \mathrm{~g}$; solid deposit on Celite $\AA$; $\lambda_{\text {detection }}$ ( $(254 \mathrm{~nm}, 280 \mathrm{~nm})$; $\mathrm{CH}_{2} \mathrm{Cl}_{2}$ in light petroleum with a gradient $0-30 \% . \mathrm{mp}=133{ }^{\circ} \mathrm{C} .{ }^{1} \mathrm{H}$ NMR $(300 \mathrm{MHz}, \mathrm{Chloroform}-\mathrm{d}) \delta=$ 2.31-2.32 (s 3H), 2.12-2.13 (s, 3H).

\section{Crystallization of $[(E)-1](T C N Q)$}

Single crystals were prepared by the co-sublimation method. To opposite ends of a $30 \mathrm{~cm}$ thin-necked glass tube, $(Z, E)-\mathbf{1}$ and TCNQ were placed. The glass tube was then sealed in vacuo with a pressure of $1 \times 10^{-5}$ mbar. In a two-zone furnace, the segments of the tube were heated to 130 and $250^{\circ} \mathrm{C}$ for the donor and the acceptor, respectively, to which the temperature was gradually raised from room temperature at a rate of $0.04{ }^{\circ} \mathrm{C} / \mathrm{min}$. The temperature of the central zone was approximately $180^{\circ} \mathrm{C}$. After three weeks, black crystals grow near the center of the tube. Since a single central zone of crystals composed of a charge transfer salt was obtained, other complexes with $(Z)-\mathbf{1}$ or different compositions were not obtained by this method. Note that our co-sublimation condition did not allow all vaporized donor and acceptor molecules flew to the central zone to react. Accordingly, there was also the deposition of each starting material along the tube, orange needle-shaped crystals of the donor molecules and yellow flake-shaped crystals of the acceptor molecules.

\section{X-ray structure determination}


Single crystals of $\left(E-\mathrm{SMe}_{2} \mathrm{Me}_{2} \mathrm{TTF}\right)(\mathrm{TCNQ})$ that are suitable for X-ray analysis were coated with Paratone$\mathrm{N}$ oil and mounted on a MicroMounts ${ }^{\mathrm{TM}}$ rod. The crystallographic data were collected on a Bruker D8 venture diffractometer equipped with MoK $\alpha$ microsource and PHOTON100 detector at the Centre de Diffractométrie (CDIFX, Université de Rennes 1, France) at $150 \mathrm{~K}$. Raw data were integrated and corrected for Lorentz and polarization effects using Bruker APEX3 v. 2015. ${ }^{24}$ The structure was solved by direct methods using the SIR92 program, ${ }^{25}$ and then refined with full-matrix least-square methods based on $F^{2}$ $\left(\right.$ SHELXL-2014) ${ }^{26}$ with the aid of the WINGX program. ${ }^{27}$ All non-hydrogen atoms were refined with anisotropic atomic displacement parameters.

\section{Electrical resistivity and thermopower}

Electrical resistivity was measured by the four-probe method down to $2.7 \mathrm{~K}$. Four gold wires $(15 \mu \mathrm{m}$ diameter) were attached to a crystal using carbon paste. Low-frequency AC current was applied to the outer wires and the resistance was estimated from the voltage measured at the inside wires. Pressure was applied to a crystal by a conventional clamp cell with an oil Daphne $\# 7373$ as a pressure medium. ${ }^{28}$ The absolute value of the pressure was estimated from the resistance change of a manganin wire at room temperature. Results for $9.1 \mathrm{kbar}$ to $14.9 \mathrm{kbar}$ are taken using a different sample from that for 1 bar to $7.4 \mathrm{kbar}$. For thermopower measurement, a crystal was attached to two copper blocks via gold foils using carbon paste. The copper blocks were alternately heated by glued tip resistors to generate $\Delta T$ less than $1 \mathrm{~K} . \Delta T$ was measured by an AuFe-chromel thermocouple. Thermopower was estimated from the slope of the generated voltage $\Delta V$ vs. the temperature difference $\Delta T .^{29}$ All measurements were conducted along the $a$-axis.

\section{ESR measurement}

ESR measurements were carried out for a single crystal using X-band spectrometer (JEOL JES-TE100). The $g$-values were calibrated by the $3^{\text {rd }}(g=2.034)$ and $4^{\text {th }}(g=1.981)$ signals of a $\mathrm{Mn}^{2+}$ marker. The crystal was located so as to make the long axis parallel to the magnetic field, and rotated by $15^{\circ}$ steps up to $180^{\circ}$ for the angle dependence measurements (Figure S5). Although the crystal was chosen carefully, the observed signal was composed of two Lorentzians when the crystal long axis was not parallel to the magnetic field. The signal was analyzed by a sum of two Lorentzians,

$$
\mathrm{f}(H)=-\frac{16 I_{a}\left(\frac{H-H_{a}^{0}}{\Delta H_{a}^{p p} / 2}\right)}{\left(3+\left(\frac{H-H_{a}^{0}}{\Delta H_{a}^{p p} / 2}\right)^{2}\right)^{2}}-\frac{16 I_{a}\left(\frac{H-H_{b}^{0}}{\Delta H_{b}^{p p} / 2}\right)}{\left(3+\left(\frac{H-H_{b}^{0}}{\Delta H_{b}^{p} / 2}\right)^{2}\right)^{2}}
$$

where $H$ was the magnetic field, $I$ was the intensity of the signal, $H^{0}$ was a center of the signal, and $\Delta H^{\mathrm{pp}}$ was the linewidth of the signal. Temperature dependence measurements were carried out down to $4.3 \mathrm{~K}$ for the single Lorentzian and double Lorentzians.

\section{Computational details}


The density functional theory (DFT) calculations were performed using a numerical atomic orbitals DFT ${ }^{30}$ approach developed for efficient calculations in large systems and implemented in the SIESTA code. ${ }^{31}$ The generalized gradient approximation to DFT and, in particular, the functional of Perdew, Burke, and Ernzerhof $(\mathrm{PBE})^{32}$ has been used. Recently ${ }^{22}$ we have found that inclusion of a Hubbard correction term ${ }^{20}$ $\mathrm{U}$ for S $3 p$ orbitals is needed for accurately describing the electronic structure of TTF-TCNQ and related two-chain molecular solids for which accurate experimental information on the bandwidth and charge transfer is available. In the present work, we have considered the dependence of the experimentally observed charge transfer for several sulfur-containing two-chain salts and found that a good agreement is found for $\mathrm{U}=6 \mathrm{eV}$. Thus, except otherwise stated we have used this value in our calculations. The contribution of core electrons has been described by norm-conserving Troullier-Martins relativistic pseudo-potentials, ${ }^{33}$ factorized in the Kleinman-Bylander form. ${ }^{34}$ Valence electrons were treated explicitly using a split-valence basis set of double- $\xi$ plus polarization functions obtained with an energy shift of 10 $\mathrm{meV} .{ }^{35}$ The energy cutoff for the real space integration mesh was set to $300 \mathrm{Ry}$ and the Brillouin zone was sampled using a mesh with $9 \times 25 \times 3$ k-points obtained using the method of Monkhorst and Pack. ${ }^{36}$ All calculations were performed using the experimental crystal structure.

\section{Acknowledgements}

This research was supported by the French National Research Agency Grants, ANR 16-ACHN-0007 and ANR 17-ERC3-0003, and a PhD grant (to M. Beau) from the Bretagne region. Y. Kiyota is grateful to JSPS for a research fellowship (17J05098). We thank Dr. A. Vacher (Rennes) for the preparation of $(Z, E)$ $\left(\mathrm{SCH}_{2} \mathrm{CH}_{2} \mathrm{CN}\right)_{2}(\mathrm{Me})_{2} \mathrm{TTF}$ and CDIFX-Rennes for access to X-ray diffractometer. Work in Spain was supported by MICIU (PGC2018-096955-B-C44 and PGC2018-093863-B-C22), MINECO through the Severo Ochoa (SEV-2015-0496) and Maria de Maeztu (MDM-2017-0767) Programs and Generalitat de Catalunya (2017SGR1506 and 2017SGR1289). Work in Japan was supported by JPSJ KAKENHI (Grant Number 16K13974 and 18H02044) and Takahashi Industrial and Economic Research Foundation.

\section{Notes and references}

† Crystal data for $[(E)-1](\mathrm{TCNQ}): \mathrm{C}_{22} \mathrm{H}_{16} \mathrm{~N}_{4} \mathrm{~S}_{6}, M=528.75$, triclinic, space group $P-1, T=150(2) \mathrm{K}, a=$ $3.8415(3) \AA, b=7.6270(7) \AA, c=18.9474(17) \AA, \alpha=90.790(3)^{\circ}, \quad \beta=90.292(3)^{\circ}, \gamma=93.350(3)^{\circ}, V=$ $554.13(8) \AA^{3}, \rho_{\text {calcd }}=1.584 \mathrm{~g} \mathrm{~cm}^{-1}, R_{1}\left(w R_{2}\right)=0.0377(0.0905)$ and $\mathrm{S}=1.133$ for 2519 reflections and 2217 reflections with $I>2 \sigma(I)$. CCDC 1901937.

1 (a) H. Akamatu, H. Inokuchi and M. Matsunaga, Nature, 1954, 173, 168. (b) C. K. Chiang, C. R. Fincher, Jr, Y. W. Park, A. J. Heeger, H. Shirakawa, E. J. Louis, S. C. Gau, and A. G. MacDiarmid, Phys. 
Rev. Lett., 1977, 39, 1098. (c) R. G. Kepler, P. E. Bierstedt and R. E. Merrifield, Phys. Rev. Lett., 1960, 5, 503. (d) L. R. Melby, Can. J. Chem., 1965, 43, 1448.

2 J. P. Ferraris, D. O. Cowan, V. V. Walatka, J. H. Pearlstein, J. Am. Chem. Soc., 1973, 95, 948.

3 (a) D. Jérome and H. J. Schulz, Adv. Phys., 1982, 31, 299. (b) D. Jérome, Chem. Rev., 2004, 104, 5565.

4 J.-P. Pouget, in Semiconductors and Semimetals, E. M. Conwell Ed., vol. 27 (Academic Press, New York) 1988, p. 88; (b) S. Kagoshima, H. Nagasawa and T. Sambongi, One-Dimensional Conductors, Springer, Berlin 1988.

5 (a) S. Horiuchi and R. Kumai, J. Am. Chem. Soc., 1998, 120, 7379. (b) S. Horiuchi, Y. Okimoto, R. Kumai, and Y. Tokura, Science, 2003, 299, 229. (c) J. Lieffrig, O. Jeannin, A. Frąckowiak, I. Olejniczak, R. Świetlik, S. Dahaoui, E. Aubert, E. Espinosa, P. Auban-Senzier, and M. Fourmigué, Chem. Eur. J., 2013, 19, 14804. (d) S. Horiuchi, K. Kobayashi, R. Kumai, N. Minami, F. Kagawa, and Y. Tokura, Nature Comm., $2015,6,7469$

6 (a) L. Ouahab and P. Batail, Acta Cryst., 1985, C41, 928. (b) R. A. L. Silva, A. I. S. Neves, E. B. Lopes, I. C. Santos, J. T. Coutinho, L. C. J. Pereira, C. Rovira, M. Almeida and D. Belo, Inorg. Chem., 2013, 52, 5300. (c) R. A. L. Silva, A. I. Neves, M. L. Afonso, I. C. Santos, E. B. Lopes, F. Del Pozo, R. Pfattner, M. Mas-Torrent, C. Rovira, M. Almeida and D. Belo, Eur. J. Inorg. Chem., 2013, 2440. (d) O. Jeannin, E. Canadell, P. Auban-Senzier and M. Fourmigué, Chem. Commun., 2016, 52, 308.

7 (a) T. Jigami, K. Takimiya, T. Otsubo and Y. Aso, J. Org. Chem., 1998, 63, 8865. (b) T. Higashino, Y. Akiyama, H. Kojima, T. Kawamoto and T. Mori, Crystals, 2012, 2, 1222.

8 (a) G. Saito and J. P. Ferraris, Bull. Chem. Soc. Jpn., 1980, 53, 2141. (b) J. B. Torrance, J. E. Vazquez, J. J. Mayerle, and V. Y. Lee, Phys. Rev. Lett., 1981, 46, 253. (c) G. Saito and Y. Yoshida, Bull. Chem. Soc. Jpn., 2007, 80, 1.

9 (a) A. Souizi and A. Robert, Tetrahedron, 1984, 40, 1817. (b) P. Pellon, E. Brulé, N. Bellec, K. Chamoutin and D. Lorcy, J. Chem. Soc., Perkin Trans., 2000, 1, 4409-4412. (c) N. Bellec and D. Lorcy, Tetrahedron Lett., 2001, 42, 3189-3191.

10 For details, see: (a) P. M. J. Szell, S. A. Gabriel, E. Caron-Poulin, O. Jeannin, M. Fourmigué and D. L. Bryce, Cryst. Growth Des. 2018, 18, 6227.

11 T. J. Kistenmacher, T. E. Phillips and D. O. Cowan, Acta Cryst., 1974, B30, 763.

12 T. E. Phillips, T. J. Kistenmacher, A. N. Bloch, J. P. Ferraris and D. O. Cowan, Acta Cryst., 1977, B33, 422

13 T. Mori, P. Wu, K. Imaeda, T. Enoki, H. Inokuchi, Synth. Met., 1987, 19, 545. (b) J. L. Galigné, J. M. Fabre and L. Giral, Acta Crystallogr. B, 1977, 33, 3827. (c) G. Keryer, P. Delhaes, J. Amiell, S. Flandrois and B. Tissier, Phys. Status Solidi B, 1980, 100, 251. 
(a) T. J. Kistenmacher, T. J. Emge, A. N. Bloch and D. O. Cowan, Acta Cryst., 1982, B38, 1193;

(b) K. Bechgaard, T. J. Kistenmacher, A. N. Bloch, Acta Cryst., 1977, B33, 417.

15 J. S. Chappell, A. N. Bloch, W. A. Bryden, M. Maxfield, T. O. Poehler and D. O. Cowan, J. Am. Chem. Soc., 1981, 103, 2442.

16 C. W. Chu, J. M. E. Harper and T. H. Geballe, Phys Rev. Lett., 1973, 31, 1491.

17 (a) E. M. Conwell, in Semiconductors and Semimetals, edited by Conwell, E. M., vol. 27 (Academic Press, New York) 1988, p. 215-292. (b) V. K. S. Shante, J. Phys. C: Solid State Phys., 1978, 11, 2561 .

18 In general, charge transfer donor-accepter complexes show a single Lorentzian due to the sufficiently rapid movement of the carriers. (a) Y. Tomkiewicz, B. A. Scott, L. J. Tao and R. S. Title, Phys Rev. Lett., 1974, 32, 1363. (b) C. Coulon and R. Clérac, Chem. Rev., 2004, 104, 5655.

19 C. Berthier, D. Jérome, G. Soda, C. Weyl and L. Zuppiroli, Mol. Cryst. Liq. Cryst., 1976, 32, 261.

20 S. L. Dudarev, G. A. Botton, S. Y. Savrasov, C. J. Humphreys and A. P. Sutton, Phys. Rev. B, 1998, 57, 1505.

21 (a) S. Ishibashi and M. Kohyama, Phys. Rev. B, 2000, 62, 7839. (b) M. Sing, R. Claessen, Th. Finteis, S. Hao, S. Hüfner and P. J. Blaha, Electron Spectrosc. Relat. Phenom., 2001, 114-116, 717. (c) J. Fraxedas, Y.-J. Lee, I. Jiménez, R. Gago, R. M. Nieminen, P. Ordejón and E. Canadell, Phys. Rev. B, 2003, 68, 195115.

22 P. Alemany, J.-P. Pouget and E. Canadell, EPL, 2016, 113, 27006.

23 (a) J.-P. Pouget, P. Alemany and E. Canadell, Mater. Horiz., 2018, 5, 590. (b) J.-P. Pouget, Physica B, 2015, 460, 45. (c) J.-P. Pouget, Physica B, 2012, 407, 1762.

24 APEX2, v. 2015; Bruker Analytical X-Ray Systems, Inc: Madison, WI, 2015.

25 A. Altomare, G. Cascarano, C. Giacovazzo, A. Guagliardi, M. C. Burla, G. Polidori, and M. Camalli, J. Appl. Cryst., 1994, 27, 435.

26 G. M. Sheldrick, Acta Cryst. 2015, C71, 3.

27 J. Farrugia, J. Appl. Crystallogr., 2012, 45, 849.

28 K. Murata, S. Kagoshima, S. Yasuzuka, H. Yoshino and R. Kondo, J. Phys. Soc. Jpn., 2006, 75, 051015 .

29 M. Chaikin and J. F. Kwak, Rev. Sci. Instrum., 1975, 46, 218.

30 (a) P. Hohenberg, W. Kohn, Phys. Rev., 1964, 136, B864. (b) W. Kohn and L. J. Sham, Phys. Rev., 1965 140, A1133.

31 (a) J. M. Soler, E. Artacho, J. D. Gale, A. García, J. Junquera, P. Ordejón and D. Sánchez-Portal, J. Phys.: Condens. Matter., 2002, 14, 2745. (b) E. Artacho, E. Anglada, O. Diéguez, J. D. Gale, A. García, J. Junquera, R. M. Martin, P. Ordejón, J. M. Pruneda, D. Sánchez-Portal and J. M. Soler, J. Phys. Condens. 
Matter, 2008, 20, 064208. (c) For more information on the SIESTA code visit: https://departments.icmab.es/leem/siesta/

32 J. P. Perdew, K. Burke and M. Ernzerhof, Phys. Rev. Lett., 1996, 77, 3865.

33 N. Troullier and J. L. Martins, Phys. Rev. B, 1991, 43, 1993.

34 L. Kleinman and D. M. Bylander, Phys. Rev. Lett., 1982, 48, 1425.

35 E. Artacho, D. Sánchez-Portal, P. Ordejón, A. García and J. M. Soler, Phys. Stat. Sol., 1999, 215, 809.

36 H. J. Monkhorst and J. D. Pack, Phys. Rev. B, 1976, 13, 5188. 